\title{
Time to Reperfusion, Door-to-Balloon Times, and How to Reduce Them
}

\author{
Margot M. Sherman Jollis and James G. Jollis
}

\subsection{Introduction}

Time to reperfusion and first-medical-contact-to-balloon times are key parameters in assessing efficiency of the primary PCI pathway while also being powerful predictors of outcome. This chapter discusses the role of these measures and how improvements may impact clinical outcomes.

\subsection{Does Time Matter?}

The time-dependent nature of myocardial injury following coronary artery occlusion has been consistently documented in the literature and runs congruent with clinical experience. Reimer and Jennings first documented an "ischemic wavefront" after transient ligation of the circumflex coronary artery in open-chested dogs, followed by reperfusion for 2-4 days (Fig. 21.1) [1]. Examining transmural slices of the posterior papillary muscle, they identified a subepicardial zone of ischemic but viable myocardium which is available for salvage for at least 3 and perhaps $6 \mathrm{~h}$ following circumflex occlusion in the dog. Thus, the time-critical nature of coronary artery reperfusion was established.

Numerous observational studies have concurred with this relationship. Combining data from 22 fibrinolysis trials involving 50,246 patients, Boersma and colleagues found the number of lives saved per 1000 patients treated to be

M. M. Sherman Jollis

Department of Biology, Denison University, Granville, OH, USA

J. G. Jollis $(\varangle)$

Department of Medicine, Duke University, Durham, NC, USA

e-mail: james.jollis@duke.edu 
Fig. 21.1 Progression of the wavefront of ischemic cell death [1]. All shaded areas, necrosis; dotted areas, necrotic muscle; horizontal cross hatching, microvascular injury; dashed line, anatomic border between ischemic circumflex coronary and nonischemic left anterior descending coronary arteries

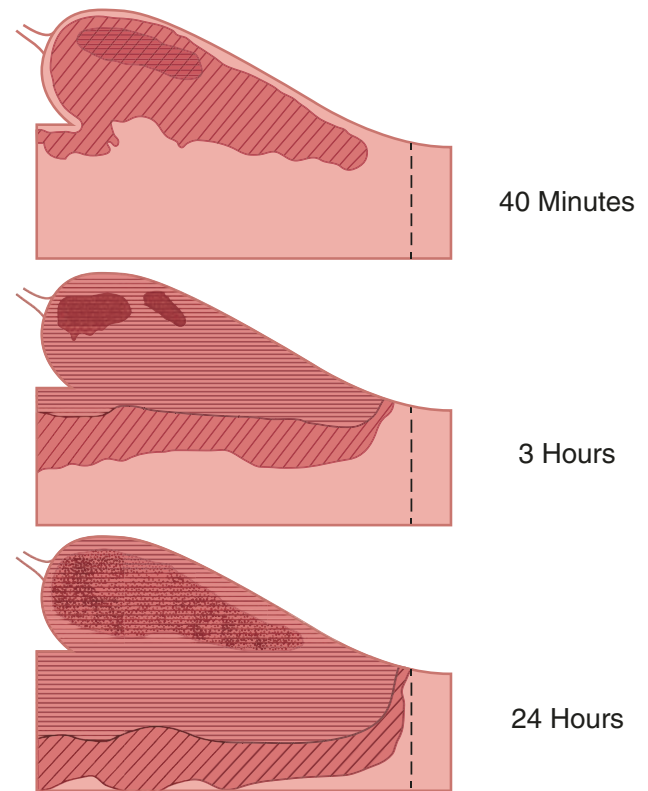

greatest for patients presenting within the first hour, with an inflection point for less benefit for patients presenting beyond $2 \mathrm{~h}$ (Fig. 21.2) [2]. This relationship between time and treatment has been documented in additional studies, with the greatest time benefit seen among patients with the highest-risk clinical presentations such as cardiogenic shock [3-5]. One notable exception to this relationship involved a manuscript by Menses et al. that showed no change in 30-day mortality for Medicare (over age 65) patients recorded in the CathPCI Registry, despite a fall in door-to-balloon (D2B) time from 83 to 67 min [6]. However, the finding was refuted in a subsequent publication that used the same data and shared two co-authors, showing that shorter patient-specific D2B times were consistently associated with lower mortality over time [7].

Most recently, data involving the coordination of ST-elevation myocardial infarction (STEMI) care in 12 regions across the United States in the Accelerator-2 project resulted in modest improvement in treatment times for patients transported by ambulance, with an increase from 67 to $74 \%$ of patients meeting the goal of first medical contact (FMC) to device within $90 \mathrm{~min}$. This improvement in treatment time was associated with a relatively large and statistically significant decline in mortality compared to patients treated at the same time without such coordination [8] (Fig. 21.3). This finding suggests that time serves as a process measure that reflects coordination of care. Likely activities involved in such coordination lead to improved mortality beyond that is expected by time metrics alone such as better paramedic training and enhanced ability of emergency physicians to move the sickest patients to coronary intervention quickly (Fig. 21.3). 


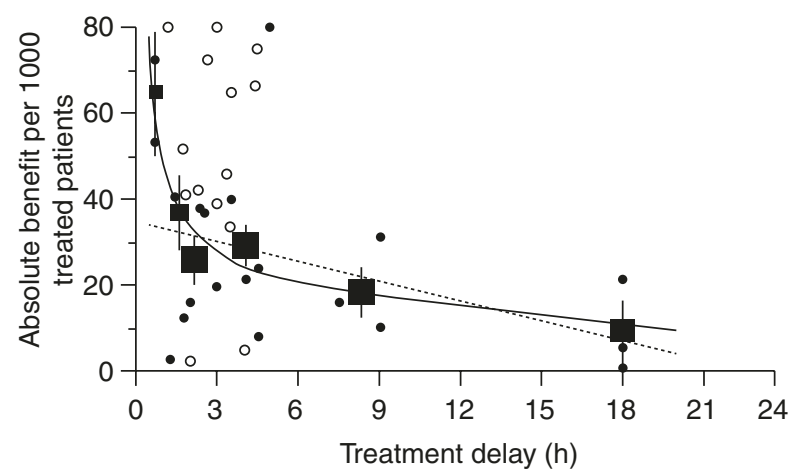

Fig. 21.2 A 35-day mortality reduction versus treatment delay [2]. Small closed dots, information from trials included in FTT analysis; open dots, information from additional trials; small squares, data beyond scale of $x / y$ cross. The linear $(34 \cdot 7-1 \cdot 6 x)$ and nonlinear $(19 \cdot 4-0 \cdot 6 x+29 \cdot 3 x 1)$ regression lines are fitted within these data, weighted by inverse of the variance of the absolute benefit in each datapoint. Black squares, average effects in six time-to-treatment groups (areas of squares inversely proportional to variance of absolute benefit described)

Fig. 21.3 In-hospital mortality according to hospital participation in Accelerator-2 with overall improvements in timely treatment versus hospitals not involved in the project without significant improvement in treatment times [8]

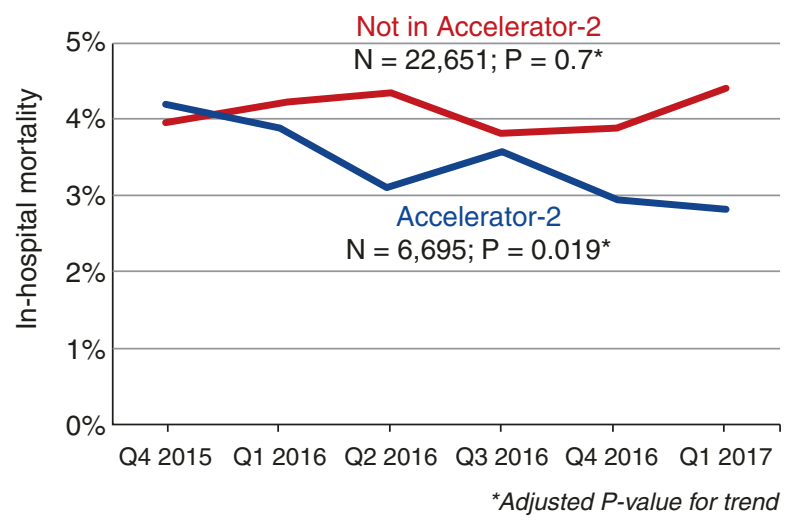

\subsection{How to Reduce Time}

The overall goal of efforts to expedite coronary reperfusion is to shorten the time from artery occlusion to resumption of normal flow. Initial efforts in the United States concentrated on a "door-to-needle" time of less than $30 \mathrm{~min}$ for patients treated with fibrinolysis and a "door-to-balloon" time of less than $90 \mathrm{~min}$. Recognizing that treatment times could be accelerated with involvement of emergency medical services (EMS) and transferring hospitals, the standard was moved to an earlier starting point, "first medical contact," maintaining the same overall goal of $90 \mathrm{~min}$. In the case of EMS-transported patients, FMC represents the time paramedics are "eyeball to eyeball" with the patient, and in the case of 
inter-hospital transfer, FMC is the time the patient arrives at the first hospital. To further encompass emergency response and treat patients sooner, the Los Angeles STEMI system starts the reperfusion clock at EMS dispatch time, and Dallas starts with symptom onset to arterial reperfusion (SOAR) time. The earlier processes are included in systematic measurements, the greater opportunity for process improvement.

In order to expedite coronary reperfusion, one needs to take a systemic survey of existing patterns of care, ideally considered separately according to the point of entry of the patient. Generally, patients present by one of four scenarios: walking into an emergency department, arriving by ambulance, transferring from another hospital emergency department, or experiencing a STEMI while hospitalized for some other condition. The latter group represents one of the most challenging with the highest mortality. Below, we will discuss approaches to accelerating coronary reperfusion according to each of these four presentation scenarios. Other tenets to improving care include data collection and feedback on a prompt and ongoing basis; establishing treatment plans agreed upon by all participating providers; establishing and maintaining treatment systems with the aid of dedicated coordinators; working across all disciplines involved in the diagnosis and treatment of acute myocardial infarction; developing as broad of a network as possible with the inclusion of competing hospitals, physician groups, and EMS agencies within a region; and recognizing that most opportunities, barriers, and solutions vary by region and can be overcome with local expertise and solutions. Our approach to organizing and transforming myocardial infarction care is summarized in Fig. 21.4. We first develop leadership, establish funding, and implement a data system. Leadership is best composed of passionate healthcare providers that span multiple disciplines and institutions. Funding is reserved to support regional coordinators, data analyses and feedback, and local and regional meetings. Once

Approach to organizing regional emergency cardiovascular care system

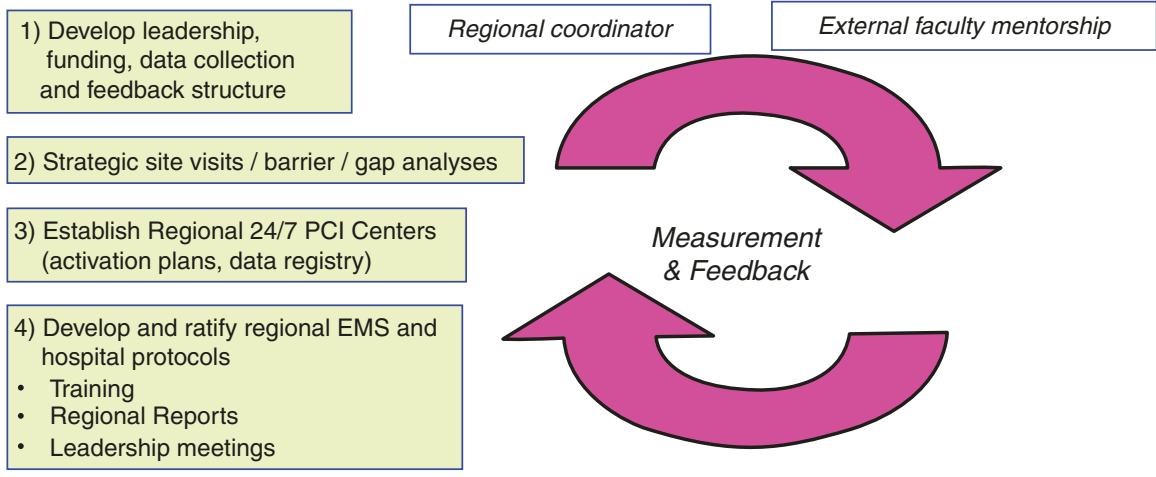

Fig. 21.4 Approach to organizing regional emergency cardiovascular care system [8] 


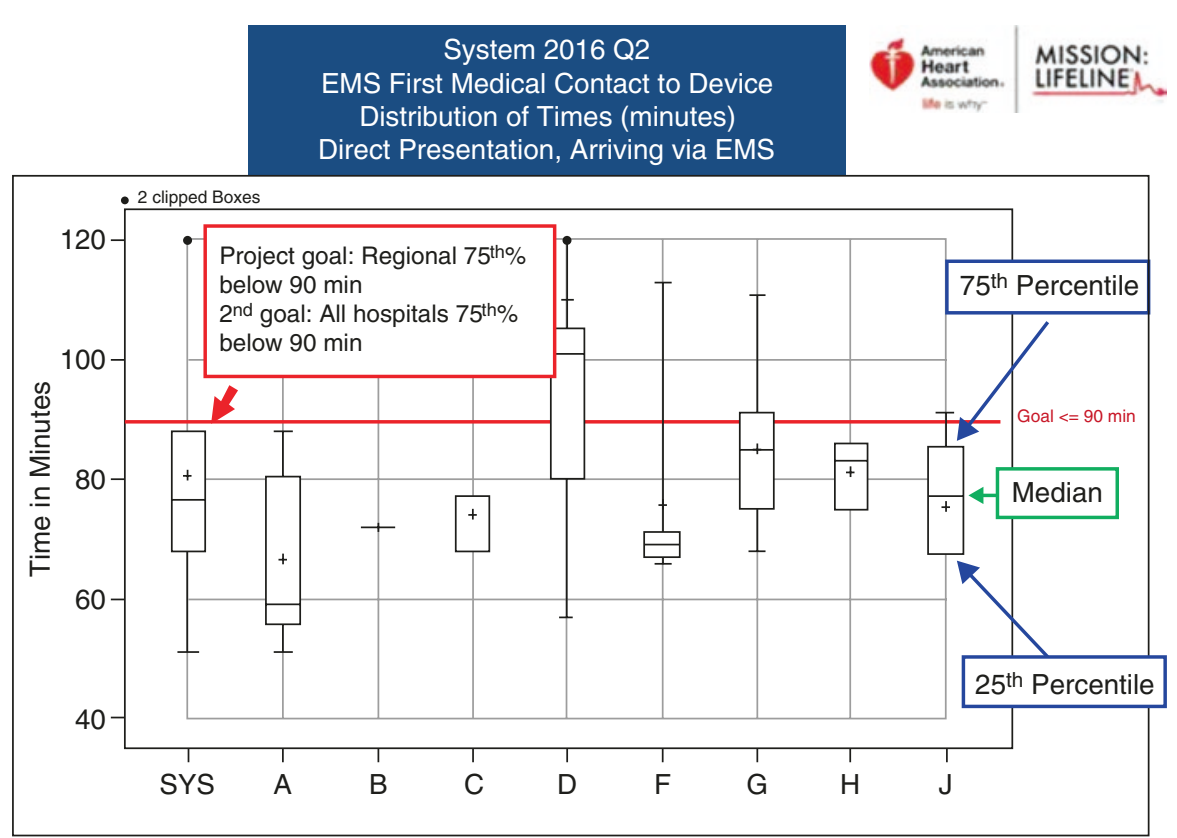

Transferred from outside facility $=$ No; Means transport to first facility $=$ Ambulance; First EKG obtained $=$ Pre-hospital or after arrival ${ }^{*} 32$

Fig. 21.5 Mission: lifeline regional report example. De-identified example of quarterly mission: Lifeline regional report used in system measurement and feedback to hospitals and emergency medical services. Bar and whisker plots. Whiskers represent ranges; bars represent interquartile range. Left hand bar is summary for region. Bars to right represent individual letter-coded hospitals. Goal of system is for each hospital to have $75 \%$ of EMS-transported patient treated within $90 \mathrm{~min}$, depicted by the top of the bar graph lying at or below the 90 min red line goal [8]

these are established, our leadership team assesses the current system for diagnosing and treating STEMI with particular attention to existing barriers that delay care. Primary PCI centers within a region are identified according to the following criteria: provide full-time primary coronary intervention, allow for single-call catheterization laboratory activation by trained paramedics or emergency physicians, accept all patients regardless of bed availability, and participate in regional data collection and reporting (Fig. 21.5). These regional PCI centers and associated medical professionals including those who refer patients to these institutions establish regional treatment protocols that are disseminating in training sessions and supported by ongoing measurement and regular meetings. An example of a common protocol or "operations manual" can be viewed at https://duke.box. com/s/ks6ipcc262illo8jyethbst8bblqybcj. We have found that systems development can be expedited through assistance from experienced colleagues from other institutions who do not have any direct relationships with the local healthcare system and can be viewed as neutral advisors, termed "external faculty" in our organizational framework. 


\subsection{Expediting Care According to Point of Entry}

Each of the four entry scenarios described above requires solutions specific to each setting. While each system and setting has unique challenges and opportunities, there are a number of common approaches that have been identified and are listed below, organized by point of entry (Table 21.1). Many of these approaches have been demonstrated to expedite treatment. For example, Fordyce showed significantly faster treatment times for hospitals that adopted paramedic catheterization lab pre-activation, single-call hospital transfer protocols, and emergency department bypass when the lab was prepared to accept an anticipated patient on hospital arrival [9]. In related work, Glickman demonstrated sequentially faster "door-in, door-out" times according to the number of eight recommended processes implemented by transferring hospitals [10]. Our work has associated paramedic prehospital activation with both faster treatment times and substantially lower mortality [10] (Fig. 21.6).

Table 21.1 Processes and protocols to expedite coronary artery reperfusion according to point of patient entry $[8,10]$

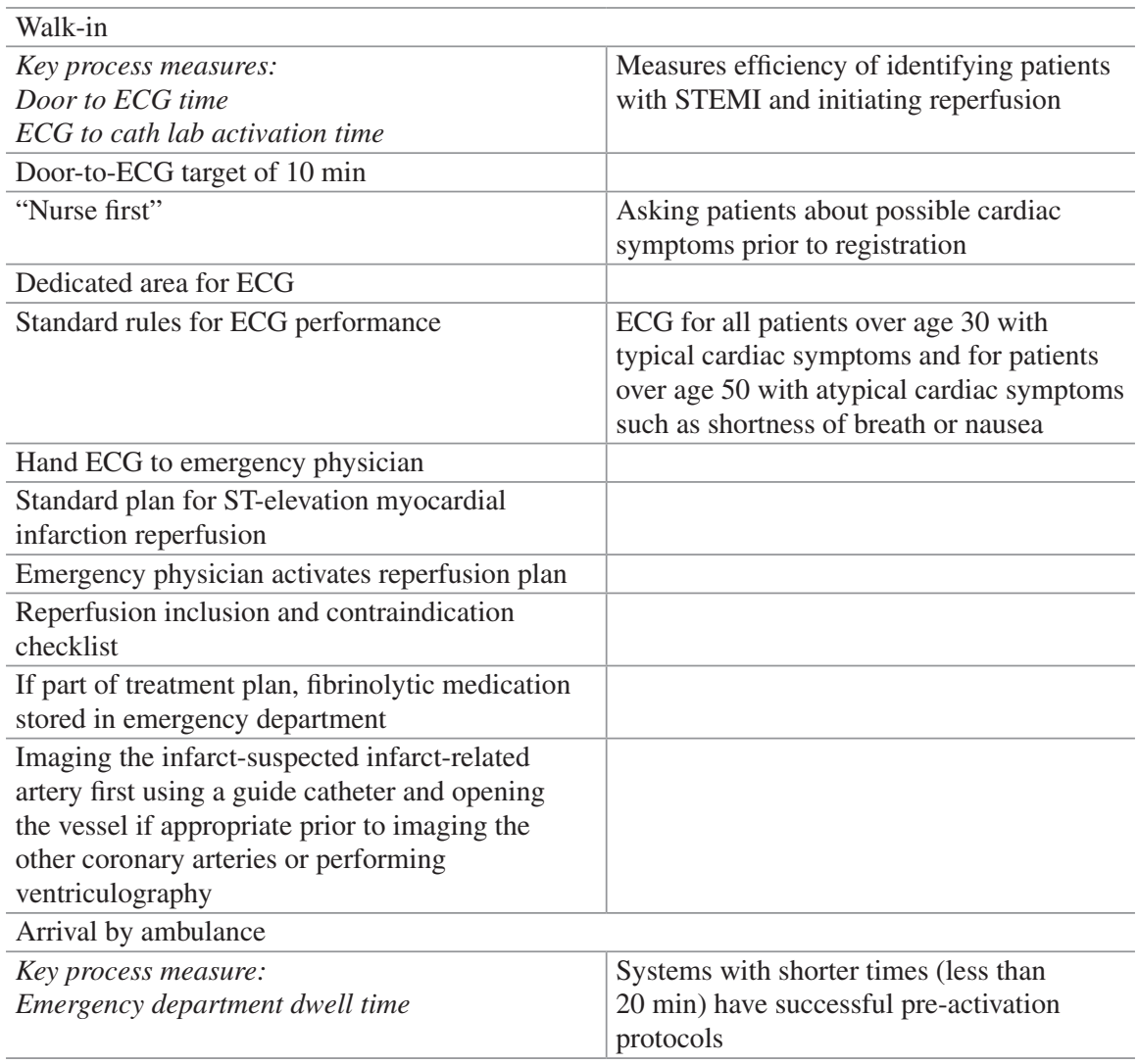


Table 21.1 (continued)

Medical leadership

Standard plan for ST-elevation myocardial infarction reperfusion

This plan should include symptom, ECG, and exclusion criteria for catheterization lab activation by paramedics. Activation should have a regionally recognized designated term such as "code STEMI" or "level 1 heart alert"

Dispatch of 12-lead ECG and personnel trained to diagnose STEMI to patients with cardiac symptoms

Standard rules for ECG performance

EMS personnel perform and read ECG

Single-call catheterization lab activation

15 min scene time

Diversion of STEMI patients directly to full-time

PCI hospitals if additional transport time less than 30-45 min.

\begin{tabular}{l|l}
\hline Emergency department bypass when possible & $\begin{array}{l}\text { Proceed directly to the catheterization } \\
\text { laboratory if staffed and ready }\end{array}$ \\
\hline
\end{tabular}

Training of paramedics on diagnosis and treatment of STEMI

Feedback within $24 \mathrm{~h}$ to all involved personnel including treatment times, catheterization findings, and patient outcomes

Health information privacy rules in the United States allow for exchange of patient-identified data between entities caring for the same patients and accrediting organizations requiring such communication

Regular interdisciplinary meetings to review STEMI cases

Inter-hospital transfer

\begin{tabular}{l|l}
\hline Key process measure: & Systems with shorter times (less than
\end{tabular}

Door-in door-out time from first hospital $40 \mathrm{~min}$ ) have successful protocols for

Similar emergency department processes to those described for "walk-in" patients above diagnosis and transfer of patients

Nurse first, ECG area, ECG rules, immediate availability of technician, handing tracing to emergency physician, emergency physician activating reperfusion system

Standard plan for ST-elevation myocardial infarction reperfusion

120 min from first hospital door threshold PCI reliably available within $120 \mathrm{~min}$, transfer to PCI hospital

PCI not reliably available within $120 \mathrm{~min}$ fibrinolysis preferred

\begin{tabular}{l|l}
\hline Nursing-led reperfusion protocols & \\
\hline Use local ambulance for transfer when available & \\
\hline Identify a primary and backup method of transfer & \\
\hline Leave the patient on the stretcher & $\begin{array}{l}\text { Patients with likely STEMI should be } \\
\text { evaluated on the stretcher and rapidly } \\
\text { moved back to the ambulance for transfer } \\
\text { when appropriate }\end{array}$ \\
\hline
\end{tabular}


Table 21.1 (continued)

Avoid intravenous drips

Aspirin, heparin bolus, and topical nitrates should be sufficient. Platelet inhibitors such as ticagrelor or cangrelor can be added according to local and regional preferences

Do not delay transfer for copying of medical records Records can be transmitted by facsimile to the receiving hospital after patient departure

\begin{tabular}{l|l}
\hline Single-call catheterization lab activation & \\
\hline Feedback within $24 \mathrm{~h}$ to all involved personnel & \\
including treatment times, catheterization & \\
findings, and patient outcomes & \\
\hline Regular interdisciplinary meetings to review & \\
STEMI cases & \\
\hline STEMI in patient already hospitalized for another condition
\end{tabular}

STEMI in patient already hospitalized for another condition

Key process measure:

Rapid response team can diagnose STEMI and activate reperfusion protocol

Standard plan for ST-elevation myocardial infarction reperfusion

Rapid-response team responds to patients with acute cardiac symptoms and trained to recognize and diagnose

Nurse leader or hospitalist authorized to activate reperfusion plan

In hospitals without full-time PCI facilities, transfer to PCI hospital for patients with contraindications to fibrinolysis such as recent surgery, gastrointestinal bleeding, or recent non-compressible arterial access site Feedback within $24 \mathrm{~h}$ to all involved personnel including treatment times, catheterization findings, and patient outcomes

Regular interdisciplinary meetings to review STEMI cases

Fig. 21.6 First-medicalcontact time to catheterization-activation time and in-hospital mortality [8]

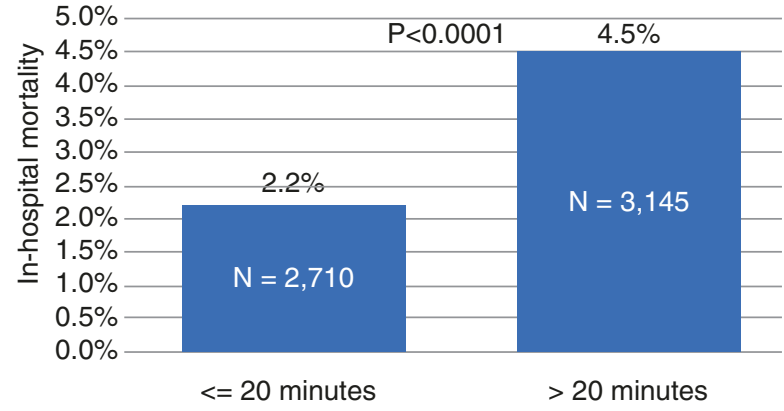




\subsection{Walk-In}

Identifying and treating STEMI patients in the emergency department has been the focus of coordinated care efforts for decades, starting with the "door-to-needle" focus of the National Registry of Myocardial Infarction and culminating in the "door-to-balloon" projects of the last decade [11]. Approximately 2 in 100 patients presenting to an emergency department with chest pain are having an acute myocardial infarction, and the process mirrors that of "finding a needle in a haystack" in often crowded emergency departments. Successful approaches have included a target door-to-electrocardiogram (ECG) time of $10 \mathrm{~min}$, asking patients about possible cardiac symptoms prior to registration, maintaining a dedicated area for rapid ECG performance, having standard rules for when to obtain an ECG, handing the ECG to the emergency physician, maintaining a standard approach to urgent coronary reperfusion most suitable to local resources and expertise, and allowing emergency physicians to activate the reperfusion plan, sometimes described as pressing the "easy button." In the catheterization laboratory, reperfusion can be accelerated by imaging the infarct-suspected infarctrelated artery first using a guide catheter and opening the vessel if appropriate prior to imaging the other coronary arteries or performing ventriculography. The trade-off of treating the infarct artery first is that important lesions in other vessels may not be taken into account prior to intervention, and the rare patient with a mechanical complication such as ventricular septal rupture may undergo coronary intervention prior to urgent surgery. Using these approaches, most patients can be treated within 30 min of hospital arrival with fibrinolysis and within 60 min with primary angioplasty.

\subsection{Arrival by Ambulance}

Likely the ideal and most expedient process for rapid diagnosis and treatment of STEMI involves patient transport by ambulance. This process includes EMS dispatch, diagnosis at the patient location by symptoms and ECG, and pre-activation of the cardiac catheterization laboratory prior to hospital arrival. In the United States, most major systems dispatch emergency technicians with advanced levels of training termed "paramedics" to patients with cardiac symptoms. These paramedics perform a 12-lead ECG on scene, interpret the tracings, and activate cardiac catheterization labs prior to the hospital using prespecified terminology such as "STEMI alert," "code STEMI," or "level 1 heart alert." The process expedites care, and in ideal instances, infarct-related arteries are opened within $1 \mathrm{~h}$ of activating the emergency medical system, "aborting" a myocardial infarction before significant damage ensues. The implementation of pre-activation involves a number of supporting processes. Most importantly, strong medical leadership from emergency medicine or interventional cardiology is required to support paramedics and maintain a system of accurate diagnoses. Prior to initiation of prehospital 
activation, cardiologists and emergency medical leadership should agree to a common threshold of symptoms, ECG findings, and exclusions to activate. A reasonable approach differentiates a "definite" from a "possible" STEMI, reserving the former for activation and the latter for additional consultation with emergency medicine and cardiology, ideally supported with ECG transmission. Systems vary in ECG criteria for activation, from $1 \mathrm{~mm}$ of ST elevation in two contiguous leads to requiring $2 \mathrm{~mm}$ of ST elevation in precordial leads. Some systems include additional support from machine interpretation, with vendor-specific statements like "meets STEMI criteria" or "acute MI suspected" resulting in lab activation. In well-performing systems, cancellation rates of $20 \%$ are reasonable and can be lowered to $10-15 \%$ with focused paramedic training and ongoing feedback. Additional processes include standard rules for ECG performance similar to the emergency department, limiting "ambulance scene time" to 15 min with a "load and go" focus, the ability of medics to direct patients to hospitals that perform percutaneous coronary intervention (PCI) on a 24-h basis, an expectation that catheterization laboratories will be ready to begin procedures within $30 \mathrm{~min}$ or less of activation, and feedback to all involved personnel within $24 \mathrm{~h}$ and at regular multidisciplinary meetings to review cases. In the best situation, when a catheterization laboratory is fully staffed and ready to accept patients on hospital arrival, the emergency department is bypassed rather than required to partake in patient registration as they pass quickly through the area. Similar to patients with major trauma going directly to an awaiting operating room or women in childbirth proceeding directly to labor and delivery, there is no mandate for STEMI patients to be evaluated in the emergency department if they can be treated immediately in the catheterization lab. The emergency department remains an important component of rapid reperfusion systems, holding patients who arrive before the lab is ready and assisting in triage of questionable cases. Bagai found that emergency department bypass significantly lowered FMC-to-device times by $20 \mathrm{~min}$ [12].

\subsection{Inter-Hospital Transfer}

Timely treatment of patients requiring inter-hospital transfer remains one of the most challenging scenarios, with almost half of patients treated beyond $2 \mathrm{~h}$ of first hospital arrival. The delays are attributable to geographic challenges and the difficulty of coordinating patient care in referring hospitals that are often small, rural, and challenged with busy emergency departments. The same tenets that assist the treatment of patients who walk into PCI hospitals apply to emergency departments that must transfer patients for treatment including a "nurse first" query of symptoms before registration and a dedicated ECG area and personnel who are immediately available to obtain ECGs, handing the tracing to the emergency physician and relying on the emergency physician to activate the coronary reperfusion plan without cardiology consultation for obvious cases. As smaller hospitals may staff their emergency departments with itinerant emergency physicians, the system and plan for treatment should heavily rely on nursing leadership and guidance. Prespecified 
reperfusion plans should be developed with the input of local experts and be guided by available resources and geography. For locations where patients can be reliably transferred for PCI within 120 min to the hospital, transferring all patients for PCI is a reasonable approach. In our experience in published data, on average, ground transport by local ambulance is as fast as helicopter transport at any distance, and ground transport is not subject to weather restrictions often faced in air travel. Effective ground transport requires the willingness of local emergency medical or critical-care transport services to convey patients to PCI hospitals, a system for rapid dispatch of transport such as the use of terms "code STEMI patient" to prioritize ambulance resources, avoiding intravenous drips with a reliance on aspirin, heparin bolus, and topical nitrates during transfer, obtaining "mutual aid" from neighboring EMS when patient transport will leave large geographies without emergency responders, and transferring patients before records and Emergency Medical Treatment and Active Labor Act (EMTALA) forms are copied with routine facsimile of records to the receiving cath lab after the patient leaves the first hospital. Having two transport alternatives for transfer patients avoids unanticipated delays. For patients arriving at the first hospital by ambulance who appear likely to have a STEMI, leaving the patient on the stretcher for ED evaluation will preclude potentially long delays in procuring a second ambulance. Transfer systems are aided by EMS that will transport STEMI patients directly to PCI hospitals from the field when geographically feasible, avoiding the delays of inter-hospital transfer.

For emergency departments that cannot reliably transfer patients to PCI hospitals for device activation within $120 \mathrm{~min}$ of first hospital arrival, fibrinolysis remains an important and timely intervention. Again, protocols should be designed to provide lytics within $30 \mathrm{~min}$ of arrival to include standard criteria for STEMI diagnosis, systematic exclusion of contraindications, and the availability for fibrinolytic drugs in the emergency department. For the $20 \%$ of patients who are ineligible for fibrinolysis, and the $25 \%$ of patients who do not show signs of clinical reperfusion according to symptom relief and ST-segment elevation resolution, protocols for rapid transfer to PCI hospitals still need to be established.

\subsection{STEMI in Patients Already Hospitalized for Another Condition}

The highest-risk STEMI involves those that occur in patients who are already hospitalized for another condition. In California discharge data, Kaul and colleagues identified a threefold higher mortality for patients with inpatient-onset STEMI [13]. While some of the higher mortality may be attributed to age and comorbid illness, significant delays in the identification and treatment of these patients likely lead to worse outcomes. Recent efforts have focused on the systematic identification and treatment of these patients. Similar to other settings, a prespecified, in-hospital STEMI plan can increase treatment rates and reduce delays. In the hospital setting, "rapid-response teams" should be dispatched to patients who are possibly experiencing an acute coronary syndrome, and these teams should be trained in the 
recognition and diagnosis of STEMI. Nursing leadership or hospitalists who routinely respond to these patients should be authorized to activate the cardiac catheterization laboratory according to standard criteria and protocols. For hospitals lacking full-time PCI facilities, a plan should be in place for rapid transfer to a PCI hospital. Hospitalized patients with recent surgery, gastrointestinal bleeding, or non-compressible arterial access sites are not eligible for fibrinolysis and merit rapid transfer. In-hospital STEMI patients should be included in reporting and quality review efforts involving other STEMI settings.

\subsection{Case Reports: Time to Reperfusion, Door-to-Balloon Times, and How to Reduce Them}

Margot M. Sherman Jollis and James G. Jollis

\subsubsection{Case Study}

A 66-year-old man with the onset of chest pain while walking his dog in the evening. He called 911, paramedics were dispatched, and an anterior ST-elevation myocardial infarction was diagnosed at his house. The catheterization laboratory was activated while he was being transported to the hospital.

\subsubsection{EMS Narrative}

"A 66-year-old male lying on his couch. The patient stated he was out walking with his family dog and started to feel pressure across his chest. The patient was pale in color but not diaphoretic. He had already taken $325 \mathrm{mg}$ of aspirin prior to EMS arrival. A 12-lead ECG showed anterior-septal STEMI. The patient was given $0.4 \mathrm{mg}$ of nitroglycerine sublingually and moved to a stretcher and secured for transport to local ED per patient's choice. IV access was established, and the patient was placed on $3 \mathrm{~L} \mathrm{O}_{2}$ due to room air saturation of $94 \%$. STEMI alert to local ED and 12-lead ECG was transmitted. Upon arrival at ED, the patient had two 5-8 s runs of V-Tach that were self-corrective. Patient care was transferred to ED nursing staff and physician by verbal report, and EMS went with patient to cath lab. $100 \%$ LAD blockage noted in cath. END OF REPORT"

\subsubsection{Cardiac Catheterization Report and Images}

Once radial access was obtained with a Glidesheath Slender $6 f$ radial introducer sheath, heparin 70 units per kilogram was administered. Verapamil $3 \mathrm{mg}$ intra-arterial was infused through the sheath. A VL 3.5 guide catheter was advanced to the left main and a guiding angiogram was performed. Ticagrelor $180 \mathrm{mg}$ was administered. A $0.014,180-\mathrm{cm}$ Extra Floppy Runthrough guidewire was advanced across the left anterior descending occlusion, and TIMI 1 flow was restored. The stenosis was 
pre-dilated with a $2.5 \times 15-\mathrm{mm}$ monorail balloon. The lesion was at the bifurcation of the first diagonal. A Forte Xsupport, $185-\mathrm{cm}$ wire was placed into the diagonal to protect the side branch. A Synergy, $3.5 \times 16-\mathrm{mm}$ coronary stent was advance across the lesion. Angiography confirmed adequate coverage of the stenosis. The stent was deployed at 16 ATM and post-dilated with the same balloon to 17 ATM. An angiogram showed the proximal portion stent was slightly undersized. The stent was postdilated with a Quantum 3.75-mm balloon to 16 ATM. A final angiogram showed no residual stenosis and TIMI 3 flow into the distal vessel. Ventriculography performed after coronary angiography showed anterior and apical hypokinesis and ejection fraction of 45\% (Figs. 21.7, 21.8, 21.9, 21.10, 21.11, 21.12, 21.13, and 21.14).

Date of Service: 12/27/1 22:09

Time Code STEMI Activated: 22:01

EMS Team: W H ,KS

ED Physician: $\mathrm{A}$

ED Nurse: $R$

Cardiologist: S

Cath Lab Team: Jennifer R, Amanda H, Seth S, Michelle B

Lesion: LAD

Comments:

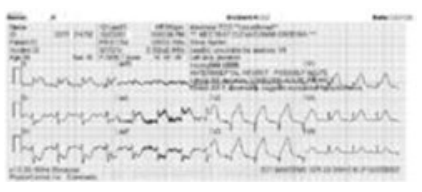

\begin{tabular}{|c|c|c|}
\hline Incident \# & $\begin{array}{c}\text { Recommended } \\
\text { Targets (mins) }\end{array}$ & Actual Date \\
\hline EMS ECG Time & 10 & $21: 49$ \\
\hline $\begin{array}{r}\text { EMS ECG Received to } \\
\text { Activation }\end{array}$ & & 2 \\
\hline EMS on Scene time & 15 & 8 \\
\hline ED Door to ECG & 10 & NA \\
\hline ECG to Activation & 5 & PTA \\
\hline Total Time in ED & 30 & 27 \\
\hline $\begin{array}{r}\text { Activation to Cath Team } \\
\text { Ready }\end{array}$ & 30 & 25 \\
\hline $\begin{array}{r}\text { Activation to MD Arrive in } \\
\text { Cath Lab }\end{array}$ & 30 & 30 \\
\hline Door to Device & 60 & 40 \\
\hline FMC to Device & 90 & 63 \\
\hline
\end{tabular}

Fig. 21.7 STEMI Feedback Report. Returned to all involved providers including EMS, emergency department, catheterization laboratory, critical care transport, and STEMI team 1 day after catheterization. Case is also reviewed in biweekly multidisciplinary team review

Fig. 21.8 Initial image, AP cranial view, radial access, guide catheter VL $3.5,100 \%$ proximal LAD, TIMI 0 flow

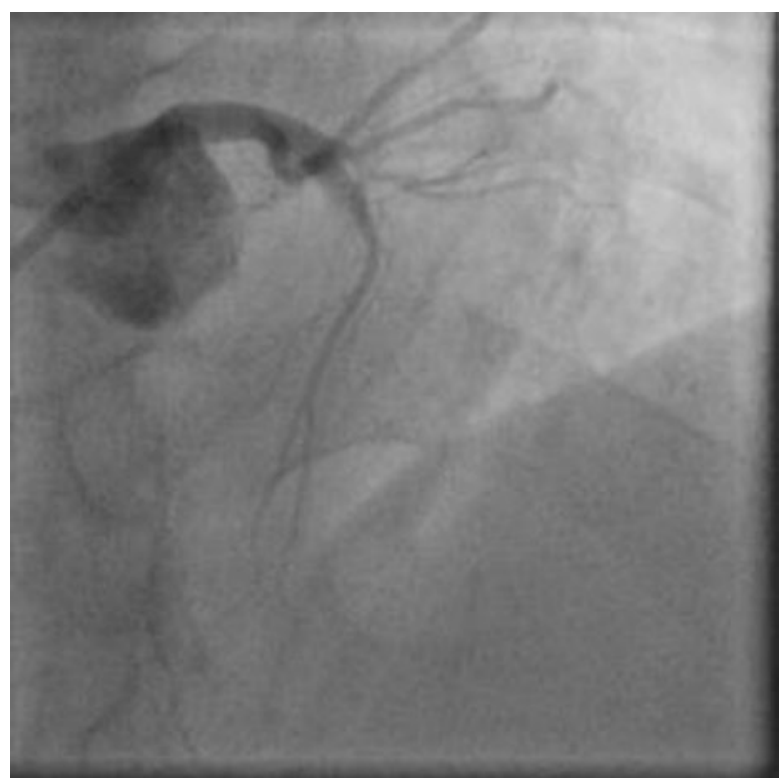


Fig. 21.9 Crossed with 0.014 guidewire and pre-dilated with a $2.5-\mathrm{mm}$ balloon

Fig. 21.10 Post initial dilation, residual filling defects, TIMI 3 flow

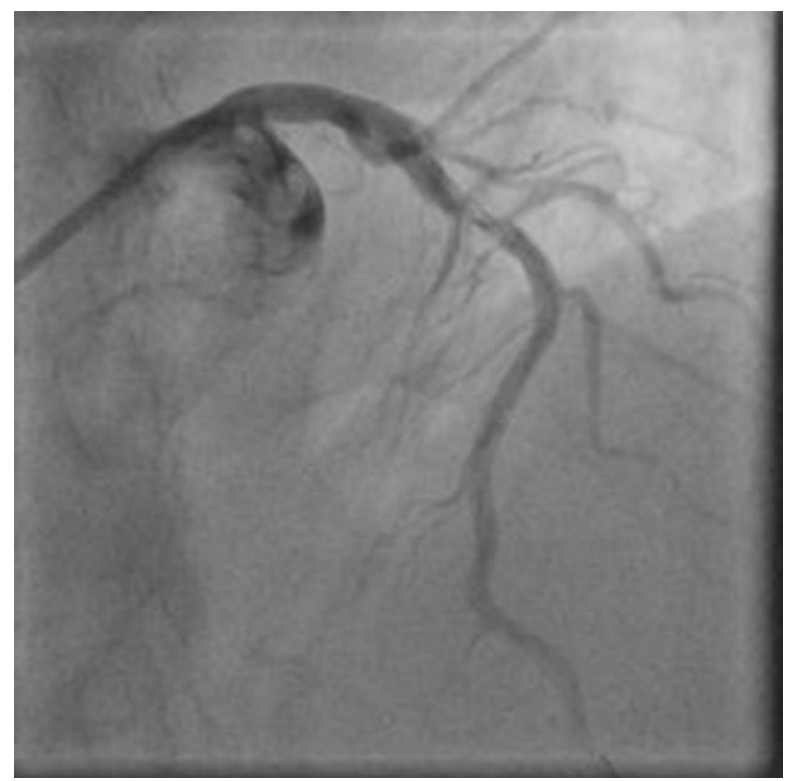


Fig. 21.11 Forte wire placed in diagonal branch for protection.

3.5 × 16-mm Synergy drug-eluting stent advanced across lesion

Fig. 21.12 Final images before wire pull. Stent deployed 16 ATM, post-dilated 17

ATM. Proximal portion post-dilated with Quantum 3.75-mm balloon to 16 ATM

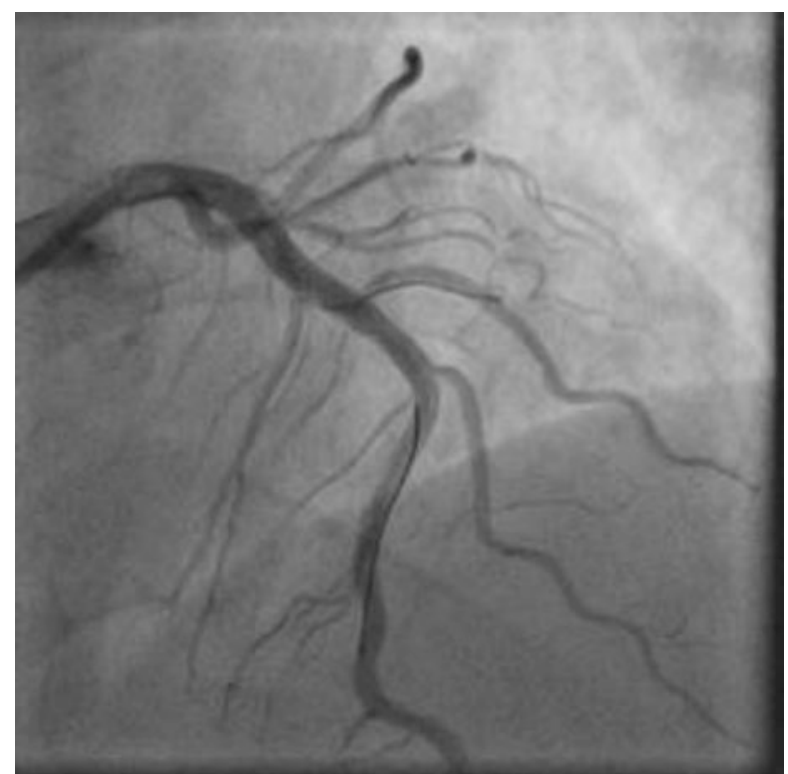


Fig. 21.13 RCA imaged after LAD opened. No stenosis

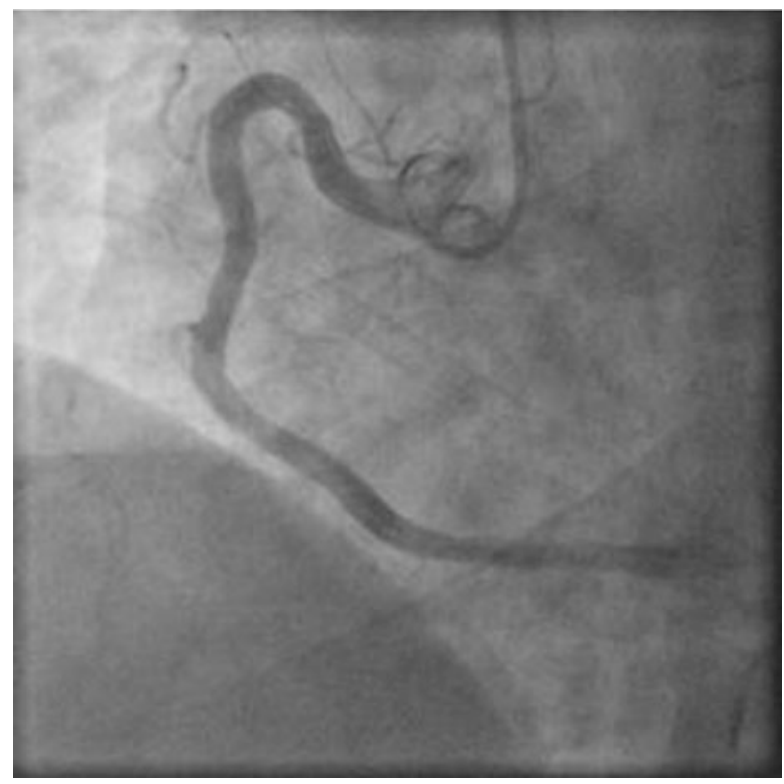

Report status:

Cumulative fluoroscopy time:

Cumulative DAP (fluoroscopy)

Cumulative DAP (exposure):

Total DAP:

Cumulative Air Kerma:

Total number of acquired runs:

Total number of acquired images:

Total number of acquired exposure images:

\section{Complete}

$7.7 \mathrm{~min}$

$17473 \mathrm{mGycm}^{2}$ $24788 \mathrm{mGycm}^{2}$

$42262 \mathrm{mGycm}^{2}$

$1,113.87 \mathrm{mGy}$

16

694

694

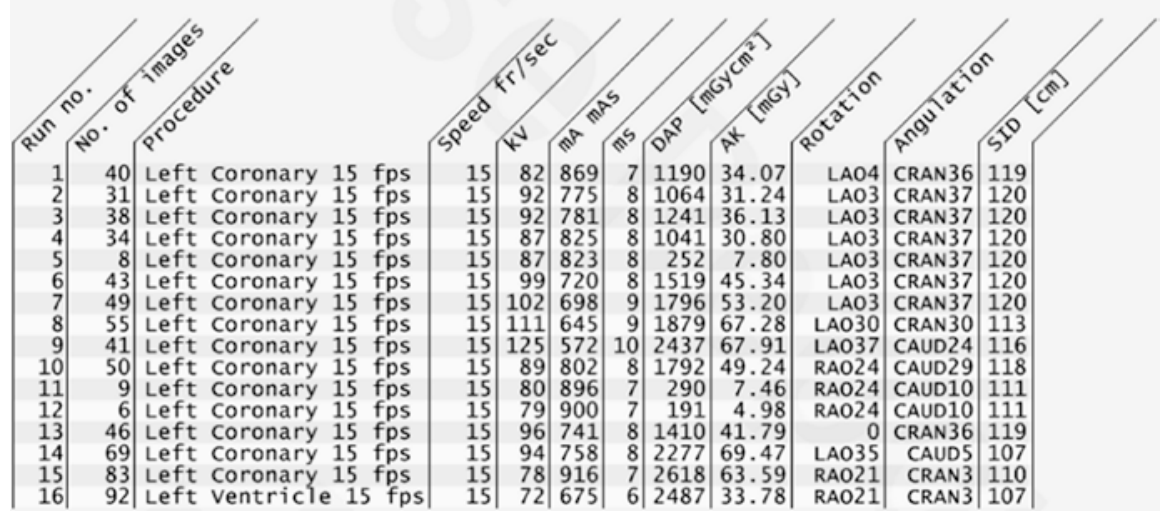

Fig. 21.14 Fluoroscopy report and camera angles 


\subsubsection{Summary}

This patient was found to have total occlusion of the left anterior descending artery with TIMI 0 flow. This was opened with a $3.5-\mathrm{mm}$ stent, and the first balloon inflation happened $63 \mathrm{~min}$ after the paramedics were "on scene" or arrived to the location of the patient. This case demonstrates how an ideal system for prehospital diagnosis and coordination between emergency medical services and hospitals serves to rapidly treat patients and thus how this translates into optimal outcomes.

\section{References}

1. Reimer KA, Lowe JE, Rasmussen MM, Jennings RB. The wavefront phenomenon of ischemic cell death. 1. Myocardial infarct size vs duration of coronary occlusion in dogs. Circulation. 1977;56:786-94.

2. Boersma E, Maas AC, Deckers JW, Simoons ML. Early thrombolytic treatment in acute myocardial infarction: reappraisal of the golden hour. Lancet. 1996;348:771-5.

3. Weaver WD. Time to thrombolytic treatment: factors affecting delay and their influence on outcome. J Am Coll Cardiol. 1995;25(Suppl 7):3S-9S.

4. Brodie BR, Stuckey TD, Muncy DB, Hansen CJ, Wall TC, Pulsipher M, Gupta N. Importance of time-to-reperfusion in patients with acute myocardial infarction with and without cardiogenic shock treated with primary percutaneous coronary intervention. Am Heart J. 2003;145:708-15.

5. Cannon CP, Gibson CM, Lambrew CT, Shoultz DA, Levy D, French WJ, Gore JM, Weaver WD, Rogers WJ, Tiefenbrunn AJ. Relationship of symptom-onset-to-balloon time and doorto-balloon time with mortality in patients undergoing angioplasty for acute myocardial infarction. JAMA. 2000;283:2941-7.

6. Menees DS, Peterson ED, Wang Y, Curtis JP, Messenger JC, Rumsfeld JS, Gurm HS. Doorto-balloon time and mortality among patients undergoing primary PCI. N Engl J Med. 2013;369:901-9.

7. Nallamothu BK, Normand S-LT, Wang Y, Hofer TP, Brush JE, Messenger JC, Bradley EH, Rumsfeld JS, Krumholz HM. Relation between door-to-balloon times and mortality after primary percutaneous coronary intervention over time: a retrospective study. Lancet. 2015;385(9973):1114-22.

8. Jollis JG, Al-Khalidi HR, Roettig ML, Berger PB, Corbett CC, Doerfler S, Fordyce CB, Henry TD, Hollowell L, Magdon-Ismail Z, Kochar A, McCarthy JJ, Monk L, O'Brien PK, Rea TD, Shavadia J, Tamis-Holland J, Wilson BH, Ziada KM, Granger CB. Impact of regionalization of ST elevation myocardial infarction care on treatment times and outcomes for emergency medical services transported patients presenting to hospitals with percutaneous coronary intervention: Mission: Lifeline Accelerator-2. Circulation. 2018;137(4):376-87. https://doi. org/10.1161/CIRCULATIONAHA.117.032446.

9. Fordyce CB, Al-Khalidi HR, Jollis JG, Roettig ML, Gu J, Bagai A, Berger PB, Corbett CC, Dauerman HL, Fox K, Garvey JL, Henry TD, Rokos IC, Sherwood MW, Wilson BH, Granger CB. Association of rapid care process implementation on reperfusion times across multiple ST-segment-elevation myocardial infarction network. Circ Cardiovasc Interv. 2017;10:e004061.

10. Glickman S, Lytle BL, Ou FS, Mears G, O'Brien S, Cairns CB, Garvey JL, Bohle DJ, Peterson $\mathrm{ED}$, Jollis JG, Granger CB. Care processes associated with quicker door-in/door-out for patients with ST-elevation myocardial infarction requiring transfer: results from a statewide regionalization program. Circ Cardiovasc Quality Outcomes. 2011;4:382-8. 
11. French WJ, Reddy VS, Barron HV. Transforming quality of care and improving outcomes after acute MI: lessons from the National Registry of myocardial infarction. JAMA. 2012;308:771-2.

12. Bagai A, Jollis JG, Dauerman HL, Peng SA, Rokos IC, Bates ER, French WJ, Granger CB, Roe MT. Emergency department bypass for ST-segment-elevation myocardial infarction patients identified with a prehospital electrocardiogram: a report from the American Heart Association mission: lifeline program. Circulation. 2013;128:352-9.

13. Kaul P, Federspiel JJ, Dai X, Stearns SC, Smith SC Jr, Yeung M, Beyhaghi H, Zhou L, Stouffer GA. Association of Inpatient vs outpatient onset of ST-elevation myocardial infarction with treatment and clinical outcomes. JAMA. 2014;312:1999-2007.

Open Access This chapter is licensed under the terms of the Creative Commons Attribution 4.0 International License (http://creativecommons.org/licenses/by/4.0/), which permits use, sharing, adaptation, distribution and reproduction in any medium or format, as long as you give appropriate credit to the original author(s) and the source, provide a link to the Creative Commons license and indicate if changes were made.

The images or other third party material in this chapter are included in the chapter's Creative Commons license, unless indicated otherwise in a credit line to the material. If material is not included in the chapter's Creative Commons license and your intended use is not permitted by statutory regulation or exceeds the permitted use, you will need to obtain permission directly from the copyright holder. 